\title{
La evaluación tradicional vs. evaluación alternativa en la FAREM-Carazo
}

MSc. Xiomara Valverde

Depto. Ciencias de la Educación y Humanidades

UNAN-MANAGUA, FAREM-CARAZO

xvalverde@unan.edu.ni

"Los métodos convencionales que utilizamos para evaluar a nuestros estudiantes no son suficientemente buenos para conseguir lo que queremos así que, necesitamos pensar radicalmente nuestras estrategias para enfrentarnos a las condiciones cambiantes de la educación superior internacionalmente" (Brown \& Glasner, 2007, p.24).

$\mathrm{E}$ n los siguientes apartados se abordará la temática relacionada con la evaluación educativa, la evaluación tradicional y alternativa, su carácter innovador, democrática y la perspectiva cultural de la misma.

La Evaluación Educativa se ha venido aplicando de forma tradicional mayormente, aunque también existe el enfoque de la evaluación alternativa que, como su nombre lo indica, ha revolucionado el enfoque tradicionalista de la misma. Esta se ha convertido en innovadora, a esto se le suma su carácter de democrática para poder cambiar su perspectiva Cultural.

En primer instancia, la evaluación educativa ha sido definida por diferentes autores usando diferentes elementos que vienen a enriquecer cada día ese concepto haciéndolo más completo e integrador. Por tanto, esta se ha aplicado en el medio escolar de una forma tradicional, esto significa que se hace a través de exámenes y pruebas escritas con el fin de obtener un número que satisfaga tanto a padres como maestros al asignar una cantidad para medir el conocimiento de sus hijos y alumnos.

Por otra parte, la evaluación educativa también presenta nuevas alternativas para evaluar el proceso enseñanza-aprendizaje, por ejemplo, el portafolio, proyectos, juegos lúdicos, entre otros. 
De igual forma, la evaluación educativa cumple con el rol de innovadora. Las estrategias de enseñanza-aprendizaje han cambiado, por lo que los procesos de la evaluación de los aprendizajes también deben transformarse para estar a tono con los procesos anteriormente mencionados.

De igual manera, la evaluación también debe ser democrática para que pueda ser innovadora, esto quiere decir, que la participación de todos los implicados en este proceso es muy importante. En el proceso de evaluación del Departamento de Ciencias de la Educación y Humanidades de la FAREM-Carazo, deben participar, desde el decano de la Facultad, la directora del Departamento, los coordinadores de carreras, los colectivos de asignaturas que están integrados por todos los docentes que imparten una asignatura en común, hasta los estudiantes, para poder contribuir de forma democrática en este proceso de cambio e innovación educativa.

Por su parte, Carbajosa (2008, pág. 8) afirma que, se busca descubrir formas de estimular a los evaluadores para que agudicen la visión de su realidad presente y comprendan las realidades de los otros, entendiendo que el proceso evaluador es una tarea compartida, en este sentido, para poder hacer una reflexión sobre las prácticas de evaluación que se han implementado en el departamento y que el resultado de las mismas tenga fruto para un cambio y optar por la innovación, la evaluación debe hacerse en conjunto, pues es una tarea de todos, no únicamente de los docentes y coordinadores de carreras.

Así mismo, para que la evaluación cumpla con la característica de innovadora tiene que estar bajo la perspectiva de lo cultural, la institución debe proporcionar las condiciones para que todo el personal involucrado en el proceso de evaluación de los aprendizajes vaya cambiando su cultura tradicional por una más innovadora.

A partir del análisis de los apartados siguientes se hará una reflexión sobre la evaluación tradicional vs. alternativa en la FAREM-Carazo.

La evaluación educativa ha sido definida por diferentes autores entre los cuales se menciona a Mora (2004) quien afirma que, la evaluación es de utilidad si va encaminada hacia el progreso donde se identifican los puntos débiles y fuertes con la idea de mejorar. Así mismo, ella expresa que es necesario tomar en cuenta la viabilidad y la equidad.

Existen muchas otras definiciones entre las cuales se tiene a:

Sanmartí (2007), quien expresa que la evaluación no solo condiciona qué se enseña, cómo se enseña, qué aprende y cómo aprende el estudiantado sino que también mide resultados. D’agostino (2007, p. 12) también afirma que, la evaluación educativa es aquel proceso orientado hacia la determinación, búsqueda y obtención de evidencias acerca del grado y nivel de calidad del aprendizaje del estudiante, para juzgar si es adecuado o no y tomar las medidas correspondientes. 
Tenbrink (2006, p. 18) menciona que la evaluación educativa, por tanto, se podría definir como un proceso de formular los juicios que se deban emitir para que tenga lugar la educación y Salinas (s.f., pág. 8) argumenta que, desde la perspectiva general, evaluar significa estimar, apreciar, calcular el valor de algo.

Como se aprecia en las definiciones, los diferentes expertos coinciden en muchos aspectos cuando se refieren a la evaluación educativa.

Por otra parte, la evaluación educativa se ha aplicado en el medio escolar de una forma tradicional, según Mateo y Martínez (2008), los enfoques tradicionales de la evaluación educativa que se han aplicado se basan en la recolecta de los datos productos de exámenes y pruebas elaboradas por el docente o la aplicación de exámenes estandarizados.

En el Modelo Educativo de UNAN-Managua (2011, p. 33) únicamente se encuentra la siguiente afirmación:

Se evalúa no solo a los estudiantes, sino todos los elementos involucrados en el proceso: los planes de estudio, los programas de asignatura, las estrategias de enseñanza-aprendizaje, los materiales utilizados, recursos físicos, el ambiente de aprendizaje y el desempeño de los docentes.

Si bien es cierto, que en las aulas de UNAN-Managua, FAREM-Carazo, en las carreras de Ciencias de la Educación, los docentes aplican una variedad de estrategias de enseñanza, lo cual lo afirman los informes de supervisiones directas elaborados por la Unidad Metodológica de esta Facultad. No se puede afirmar que los maestros al momento de evaluar los conocimientos apliquen la evaluación alternativa. Por el contrario, se aplica una forma de evaluación tradicional y básicamente sumativa, como lo afirman Bordas y Cabrera (2001) sobre las estrategias de enseñanza muy innovadoras que aplican los docentes pero que son evaluadas de manera tradicional.

Por consiguiente, se observa la contradicción ya quelas prácticas de enseñanza-evaluación no se complementan, concretamente, en las clases de gramática de la Carrera de Inglés, los docentes aplican una metodología muy activa y constructivista porque los estudiantes usan la gramática en juegos de roles, elaboran murales informativos, escriben diarios, entre otras.

Sin embargo, al momento de evaluar, el $40 \%$ que corresponde al examen escrito, los docentes elaboran extensas pruebas de selección múltiple (cuatro a cinco páginas), esto explica la contradicción de aplicar estrategias innovadoras al momento de impartir la clase pero evalúan los aprendizajes de manera tradicional.

A continuación se expone un estudio realizado por Aburto (2014) en el que asevera que entre los docentes de la UNAN-Managua, FAREM-Carazo existe la confusión entre calificación y evaluación, no saben distinguir entre qué evaluar, si contenidos o aprendizajes y tampoco saben 
distinguir cuales son los aprendizajes significativos, los que los estudiantes necesitarán poner en práctica en la vida real y laboral.

Según el estudio de Aburto (2014), los docentes de las disciplinas de Matemática, Física General, Francés II, Historia de Nicaragua, entre otras, de la FAREM-Carazo, los resultados indican que:

El $70 \%$, es decir, 14 profesores diseñaron instrumentos de evaluación, cargados con respuestas reproductivas a nivel del conocer y saber. Entre los ítems utilizados mayoritariamente pasan las preguntas de reproducción de contenidos en los que sobresalen: defina, complete, enumere, selección múltiple y de falso y verdadero.

En la práctica es evidente que los distintos actores sujetos del estudio, no dominan los procesos de Enseñanza-Aprendizaje y el proceso de Evaluación educativa.

No obstante, aunque existe el enfoque de evaluación alternativa no es muy aplicado en nuestro ámbito universitario. "Evaluación alternativa: hace referencia a todas las técnicas y métodos de evaluación que intentan superar la metodología tradicional de evaluación, basada en la simple realización de pruebas y exámenes, con la finalidad única y principal de calificar" (Ávila, Calatayud, De Miguel, Canton, Catillo, y Zaitegui, 2010, p.17).

Según Mateo y Martínez (2008), la evaluación educativa también presenta nuevas alternativas para evaluar el proceso enseñanza-aprendizaje. Este enfoque se basa en la observación directa del trabajo de los estudiantes y de sus habilidades. La evaluación alternativa también es conocida como auténtica y de ejecución. Es decir, para que la evaluación alternativa sea auténtica, los estudiantes tienen que realizar actividades que pertenecen a la vida real. Es importante señalar que este tipo de pruebas no puede ser diseñado por el profesor, en cambio, en la evaluación por ejecución, los estudiantes deben elaborar un producto, construyen, dan soluciones a problemáticas planteadas.

En relación a lo antes expuesto por Mateo y Martínez (2008) sobrela evaluación alternativa y analizando la realidad del Departamento de Ciencias de la Educación y Humanidades de la FAREM-Carazo, se puede afirmar que, los docentes de este departamento únicamente aplican la evaluación tradicional, esto es fácilmente comprobable al revisar los archivos de la dirección de este Departamento en los que los docentes presentan las propuestas de exámenes que tienen las características en un cien por ciento de tradicionales por los ítems elaborados.

Aunque el Modelo Educativo, Normativa y Metodología para la Planificación Curricular 2011 (pág. 32-33) expresa que:

“La evaluación se concibe como parte del proceso enseñanza-aprendizaje, y por tanto, su objetivo primordial no es la evaluación sumativa de los conocimientos adquiridos por los estudiantes. La evaluación se entiende como la reunión de información de forma ordenada y sistemática 
que permite la toma de decisiones que posibilitan reconducir, redefinir o bien reorientar el desarrollo del proceso educativo. La auto reflexión y reflexión sobre las causas de las deficiencias encontradas facilitan la toma de decisiones que posibilitan reconducir el proceso, y por tanto, desarrollar una evaluación formativa. Esto permite que los estudiantes conozcan cómo han avanzado y en qué lugar del proceso se encuentran, conocer sus dificultades y que puedan hacer para mejorar. Esto implica la creación de espacios, ambientes e instrumentos que favorezcan la heteroevaluación, autoevaluación y coevaluación.

A lo anteriormente expuesto por el Modelo Educativo de la UNAN-Managua, habrá que agregar los diferentes talleres que se han impartido para explicar la temática de evaluación de los aprendizajes por las Unidades correspondientes dirigidos a los directores, coordinadores de carreras y a los docentes de aula, sin embargo, los líderes no se han preocupado por cambiar el enfoque de evaluación de los aprendizajes obviando, de forma clara, las indicaciones del Modelo Educativo de la UNAN-Managua y de la existencia de la evaluación alternativa y aplicando la evaluación tradicional, que por cultura se ha aplicado por décadas.

Por otro lado, la evaluación, según Mateo y Martínez (2008), se ha convertido en innovadora, porque ha sufrido cambios en estos últimos diez años, lo cual se ha transformado en la innovación, la más significativa en el campo de la educación.

Así mismo, González y Ayarza (1996, citados por Mora, 2004) afirman que la evaluación educativa califica como un instrumento para sensibilizar el quehacer académico y facilitar la innovación. Así pues, Santamaría Vizcaíno (2005) expresa que, si el docente usa técnicas novedosas y creativas, estará enriqueciendo la magia del aprendizaje y también la evaluación, de igual forma se beneficiará con esta actitud de cambio, resultando la oportunidad para que el estudiante aprenda y demuestre lo que sabe. Señala, además, que las inteligencias múltiples, el uso del portafolio, proyectos, actividades lúdicas, entre otras, pertenecen a la evaluación cualitativa. Afirma también, que la evaluación cualitativa emplea sobre todo la observación, pero utiliza un rango más amplio de variables educativas (p.60) Pero asimismo afirma que la evaluación cuantitativa se apoya en la medición, casi como única variable educativa (p.60). Con estas afirmaciones se puede calificar a la evaluación alternativa como cualitativa.

Según Stufflebeam y Shinkfield (1995, citados por Mora, 2004) la Evaluación Iluminativa abarca la evaluación cualitativa y de acuerdo a Parlet y Hamilton (1997, citados por Mora, 2004) el objetivo principal de este modelo es la descripción y la interpretación más que la valoración y la predicción.

Por otro lado, Falchikov y Thompson (2008) afirman, en un reciente estudio de Higher Education Academy (HEA) en Inglaterra, que entre los seis tipos de innovaciones aplicadas en la evaluación de conocimientos, solo presentaron evaluaciones no escritas, presentaciones 
orales, escritos no convencionales, portafolios, evaluaciones en grupo y trabajo colaborativo, participación de los estudiantes en el proceso de evaluación y el uso de la tecnología.

Si bien es cierto, que todo lo relacionado a la innovación en la evaluación es muy interesante e importante, su aplicación enfrenta muchos retos como: la resistencia al cambio, el poco interés de asumir compromisos de participación y el miedo a enfrentar nuevos retos. El proceso de evaluación toma tiempo y debe promoverse la constante reflexión de la práctica para poder lograr un cambio de actitud por parte del profesorado, si se logra esto, la práctica de la evaluación y el proceso de enseñanza-aprendizaje mejorará (Mora, 2004).

De esta manera, una de las propuestas para ser implementadas a la brevedad en el Departamento de Ciencias de la Educación y Humanidades de la FAREM-Carazo es la inclusión de la Evaluación Alternativa para que el proceso de Evaluación de los aprendizajes sea más democrático e innovador. No es tarea fácil, sin embargo, se puede lograr un cambio si los docentes hacen una reflexión sobre sus prácticas evaluativas.

Por último, se aborda la perspectiva Cultural, que tiene mucho que ver al analizar cómo son interpretadas las innovaciones e integradas en el contexto social y cultural de una institución. Desde esta perspectiva, la innovación en la evaluación de los aprendizajes se supone deber ser aplicada en coordinación con diferentes estrategias (Mateo y Martínez, 2008).

Esta perspectiva cultural ha de ser adoptada poco a poco por los docentes de la FAREMCarazo, especialmente los que laboran en el Departamento de Ciencias de la Educación y Humanidades. La cultura de evaluación hasta ahora aplicada es la de la evaluación tradicional basada en la medición. Es un reto para los próximos tiempos y para las autoridades encargadas de la parte metodológica y técnica de esta facultad.

La cultura de la innovación en la que los estudiantes sean partícipes de su propia evaluación de los aprendizajes causa un poco de reacción negativa en los docentes que se resisten a salir de su zona de confort, sin embargo, si se aplica por etapas, esto será una realidad para mejorar el proceso de evaluación de los aprendizajes. "Introducir innovaciones en procesos de evaluación amerita seguir ciertos pasos con el fin de asegurarse metas y no conducirse a la deriva, debido a que cuando se intenta encajar cualquier cambio en la sociedad, esta reacciona ofreciendo resistencia a pesar de que lo que se persigue es mejorar o reparar situaciones problemáticas o desgastadas por el tiempo" (Arias Peñaloza y Arias Lara, 2011, p.5).

En conclusión, la evaluación educativa es democrática, alternativa, tradicional e innovadora y esta está influenciada por la cultura que se rige en una instrucción. Es pues, un reto para las personas involucradas en el proceso enseñanza-aprendizaje aportar cambios para 
mejorar en el proceso de evaluación de los aprendizajes a través de las innovaciones y nuevas alternativas en este campo de la educación.

\section{REFERENCIAS}

Aburto, P. (2014). Análisis de la evaluación educativa y su relación con la planificación docente $y$ el rendimiento académico estudiantil durante el primer semestre del año 2008. En Revista Torreón Universitario. Número 7. Pág. 12 a 22. Recuperado de: http://revistasnicaragua.net.ni/index. $\mathrm{php} /$ torreon/search/search

Arias Peñaloza, Milvia Lisette y Arias Lara, Sergio Alejandro. (2011). Evaluar los aprendizajes: un enfoque innovador. En Educere: Revista Venezolana de educación. Volumen 15. Número 51. Pág. 357 a 368. Recuperado de: http://www.redalyc.org/ pdf/356/35621559006.pdf

Ávila, M., Calatayud, de Miguel, C. Canton, I., Catillo, S. \& Zaitegui, N. (2010). La evaluación como proceso sistemático para la mejora educativa. Recuperado de: https:// books.google.com.ni/books?id=OCwbAg AAQBAJ\&pg=PA17\&dq=evaluacion + alte rnativa+concepto\&hl=es-419\&sa=X\&ved $=0$ ahUKEwj1kcjOyMDSAhVS52MKHTIF CrIQ6AEIMDAE\# $\mathrm{v}=$ onepage $\& \mathrm{q}=$ =valuac ion\%20alternativa\%20concepto\&f=false

Brown, S. \& Glasner, A. (2007). Evaluar en la universidad: problemas y nuevos enfoques.

Bordas, M. \& Cabrera, F. (2012). Estrategias de evaluación de los aprendizajes centrados en el proceso. En revista española de pedagogía. Número 218. Pág. 25 a 48. Recuperado de: $\quad$ https://dpegp.files.wordpress. com/2012/04/lectura-1-eva-apren.pdf

Carbajosa, D. (2008). Debate desde paradigmas en la evaluación educativa. Recuperado de: http://docenpostav. unan.edu.ni/pluginfile.php/3686/ mod_resource/content/0/DEBATE\%20 DESDE\%20LOS\%20PARADIGMAS\%20 E N \% 20 L A \% 20 E VA L UACION\% 20 E DUCAT IVA \% $20 \% 20$ D I A A \% 20 CARBAJOSA.pdf

D’agostino, Giusppa. (2007). Aspectos teóricos de la evaluación educacional. Recuperado de: https://books.google.com.ni/books?i $\mathrm{d}=8$ ueTibkvwtwC\&pg=PA17\&dq=definici $\%$ C3\%B3n+de+evaluaci\%C3\%B3n+educa tiva\&hl=es-419\&sa=X\&ved $=0$ ahUKEwjAj amY1cTSAhUqw1QKHaNaCp8Q6AEIHjA $\mathrm{B} \# \mathrm{v}=$ onepage\&q\&f=false

Falchikov, N. \& Thompson, K. (2008). Assessment: What Drives Innovation? Journal of University Teaching \& Learning Practice, 5(1). Disponible en: http:// ro.uow.edu.au/jutlp/vol5/iss1/5

UNAN-MANAGUA. (2011). Modelo educativo, normativa y metodología para la planificación curricular 2011. Managua.

Mateo, J. \& Martínez, Francesc. (2008). La evaluación alternativa de los aprendizajes. Recuperado de: http://www.ub.edu/ice/ 
sites/default/files/docs/qdu/3cuaderno. pdf

Mora Vargas, A I. (2004). La evaluación educativa: Concepto, períodos y modelos. Revista Electrónica "Actualidades Investigativas en Educación". Recuperado de: http://www.redalyc.org/articulo. oa?id=44740211

Salinas, Bernardino. (s.f.). Evaluación de los estudiantes en la educación superior. Recuperadode:http://www.postgradoune. edu.pe/documentos/evaluacion/La\%20 evaluacion\%20 estudiantes\%20 en $\% 20$ la\%20ESuperior\%20UV.pdf

Sanmartí, Neus. (2007).10 ideas claves. Evaluar para aprender. Recuperado de: https:// books.google.com.ni/books?id=BuAkkh RUtYgC\&printsec $=$ frontcover\&dq $=$ evalu aci\%C3\%B3n+educativa+neus+sanmarti \&hl=es-419\&sa=X\&ved=0ahUKEwi8vM n8icPSAhUOxGMKHf84Ar8Q6AEIGjAA$\# \mathrm{v}=$ onepage \&q=evaluaci\%C3\%B3n\%20 educativa\%20neus\%20sanmarti\&f=false
Santamaría Vizcaíno, Martin. (2005) ¿Cómo evaluar aprendizajes en el aula? San José, C.R. EUNED.

Tenbrink, Terry. (2006). Evaluación: Guía práctica para profesores. Recuperado de: https://books.google.com.ni/books?id=C JyeZusF6YIC\&pg=PA18\&dq=definici\%C $3 \% B 3 n+d e+$ evaluaci\%C3\%B3n+educativa \&hl=es-419\&sa=X\&ved=0ahUKEwiihs63cTSAhUHxFQKHQykBc4Q6AEIQjAI\#v= onepage\&q=definici\%C3\%B3n\%20de\%20 evaluaci\%C3\%B3n\%20educativa\&f=false 